\title{
"Black" Responses Dominate Macaque Primary Visual Cortex V1
}

\author{
Chun-I Yeh (葉俊毅), ${ }^{*}$ Dajun Xing (邢大军), ${ }^{*}$ and Robert M. Shapley \\ Center for Neural Science, New York University, New York, New York 10036
}

Achromatic visual information is transferred from the retina to the brain through two parallel channels: ON-center cells carry "white" information and OFF-center cells "black" information (Nelson et al., 1978; Schiller, 1982; Schiller et al., 1986). Responses of ON and OFF retinal and thalamic neurons are approximately equal in magnitude (Krüger and Fischer, 1975; Kremers et al., 1993), but psychophysical studies have shown that humans detect light decrements (black) better and faster than increments (white) (Blackwell, 1946; Short, 1966; Krauskopf, 1980; Whittle, 1986; Bowen et al., 1989; Chan and Tyler, 1992; Kontsevich and Tyler, 1999; Chubb and Nam, 2000; Dannemiller and Stephens, 2001). From recordings of single-cell activity in the macaque monkey's primary visual cortex (V1), we found that blackdominant neurons substantially outnumbered white-dominant neurons in the corticocortical output layers $2 / 3$, but the numbers of black- and white-dominant neurons were nearly equal in the thalamocortical input layer 4c. These results strongly suggest that the black-over-white preference is generated or greatly amplified in V1. The predominance of OFF neurons in layers $2 / 3$ of V1, which provide visual input to higher cortical areas, may explain why human subjects detect black more easily than white. Furthermore, our results agree with human EEG and fMRI findings that V1 responses to decrements are stronger than to increments, though the OFF/ON imbalance we found in layers 2/3 of macaque V1 is much larger than in the whole V1 population in the human V1 experiments (Zemon et al., 1988, 1995; Olman et al., 2008).

\section{Introduction}

Humans tend to read faster, make fewer errors and detect more mistakes when reading black-on-white texts (negative contrasts) than when reading white-on-black texts (positive contrasts) (Bauer and Cavonius, 1980; Buchner and Baumgartner, 2007). One may argue that the superiority in reading black texts is likely due to "practice", since most reading materials consist of negative-contrast texts. However, the "black" advantage also appears in many other psychophysical tasks (Blackwell, 1946; Short, 1966; Krauskopf, 1980; Whittle, 1986; Bowen et al., 1989; Chan and Tyler, 1992; Kontsevich and Tyler, 1999; Chubb and Nam, 2000; Dannemiller and Stephens, 2001; Chubb et al., 2004; Badcock et al., 2005). For instance, Blackwell (1946) reported in a classic paper that the threshold of detecting negative contrast was consistently lower than that of detecting positive contrast, Chan and Tyler (1992) and Kontsevich and Tyler (1999) reported much lower thresholds for contrast discrimination of negative-contrast versus positive-contrast targets, and Chubb and Nam (2000) found the judgments of textural contrast were primarily mediated by the visual channel detect-

\footnotetext{
Received April 27, 2009; revised June 25, 2009; accepted Aug. 7, 2009.

This work was supported by grants from the U.S. National Institutes of Health (EY-001472) and the U.S. National Science Foundation (NSF-0745253), and by fellowships from the Robert Leet and Clara Guthrie Patterson Trust and the Swartz Foundation. Thanks go to Drs. Marianne Maertens and PatrickE. Williams for their help with experiments and Drs. Siddhartha Joshi, Anita A. Disney, and Michael J. Hawken for their advice in histology.

${ }^{*}$ C.-I.Y. and D.X. contributed equally to this work.

Correspondence should be addressed to Dr. Chun-I Yeh, Center for Neural Science, New York University, 4 Washington Place, New York, NY 10003. E-mail: ciy@cns.nyu.edu.

DOI:10.1523/JNEUROSCI.1991-09.2009

Copyright $\odot 2009$ Society for Neuroscience $\quad$ 0270-6474/09/2911753-08\$15.00/0
}

ing negative contrast. Moreover, dark targets appeared more salient than bright targets for infants (Dannemiller and Stephens, 2001), implying that the advantage in perceiving negative contrasts is probably intrinsic rather than shaped by experience. This raises a question: what part of the visual pathway may be responsible for the substantial black/white imbalance in visual perception?

Signals about black and white visual information are transferred from the retina to the brain through two parallel channels: OFF-center cells carry black information and ON-center cells "white" information (Nelson et al., 1978; Schiller, 1982; Schiller et al., 1986). The ON and OFF channels converge for the first time in the primary visual cortex V1 (cf. Sherk and Horton, 1984; Reid and Alonso, 1995), which provides information to higher cortical areas that are critical for detecting boundary and shape (Pasupathy and Connor, 1999; Zhou et al., 2000). It is known that responses of $\mathrm{ON}$ and $\mathrm{OFF}$ retinal and thalamic neurons are approximately equal in magnitude (Krüger and Fischer, 1975; Kremers et al., 1993), and we report here that neurons in the V1 input layer $4 \mathrm{c}$ also have roughly balanced response magnitudes for black and white stimuli. However, we also found evidence, from recordings of single-cell activity in the macaque monkey's $\mathrm{V} 1$, that the majority of neurons in the corticocortical output layers $2 / 3$ of $\mathrm{V} 1$ showed stronger responses to black stimuli than to white stimuli. Our neurophysiological results on the preference for black in macaque V1 are consistent not only with human psychophysics but also with the findings of EEG and fMRI studies that human V1 responses to decrements are stronger than to increments (Zemon et al., 1988, 1995; Olman et al., 2008). Our major new finding is that the preference for black emerges in V1, 
in the cells of the output layers, from almost balanced input at the level of input layer 4c.

\section{Materials and Methods}

Animal preparation. Acute experiments were performed on five adult Old World monkeys (Macaca fascicularis). All surgical and experimental procedures were performed in accordance with the guidelines of the U.S. Department of Agriculture and were approved by the University Animal Welfare Committee at the New York University. Animals were sedated with midazolam $(0.3 \mathrm{mg} / \mathrm{kg}$, i.m. $)$ and anesthetized initially with ketamine $(30 \mathrm{mg} / \mathrm{kg}$, i.m.) and then with isoflurane (1.5-3.5\% in air) after being intubated. After intravenous catheters were placed in both hindlimbs, the animal was placed in a stereotaxic frame and maintained on opioid anesthetic (sufentanil citrate, $6-12 \mu \mathrm{g} \cdot \mathrm{kg}^{-1} \cdot \mathrm{h}^{-1}$, i.v.) during the craniotomy. A craniotomy $(\sim 5 \times 7 \mathrm{~mm})$ was made in one hemisphere posterior to the lunate sulcus $(\sim 15 \mathrm{~mm}$ anterior to the occipital ridge, $\sim 10 \mathrm{~mm}$ lateral from the midline). A small opening in the dura $(\sim 3 \times 5$ $\mathrm{mm}$ ) was made to provide access for multiple electrodes. After surgery, anesthesia was maintained with a continuous infusion of sufentanil citrate $\left(6-12 \mu \mathrm{g} \cdot \mathrm{kg}^{-1} \cdot \mathrm{h}^{-1}\right.$, i.v.), and the animal was paralyzed with vecuronium bromide $\left(0.1 \mathrm{mg} \cdot \mathrm{kg}^{-1} \cdot \mathrm{h}^{-1}\right.$, i.v. $)$ and maintained hydrated by giving fluid at a rate of $\sim 6 \mathrm{ml} \cdot \mathrm{kg}^{-1} \cdot \mathrm{h}^{-1}$ (the rate was adjusted hourly to keep the urine specific gravity within normal range $(\sim 1.010 \mathrm{~g} / \mathrm{ml})$ ). Vital signs, including heart rate, blood pressure, electroencephalogram, and oxygen level in blood were closely monitored throughout the experiment. Expired carbon dioxide was maintained close to $32-38 \mathrm{mmHg}$ and rectal temperature was kept at a constant $37^{\circ} \mathrm{C}$ using a feedbackcontrolled heating pad. A broad spectrum antibiotic (Bicillin, 50,000 $\mathrm{IU} / \mathrm{kg}$, i.m.) and anti-inflammatory steroid (dexamethasone, $0.5 \mathrm{mg} / \mathrm{kg}$, i.m.) were given on the first day and every $24 \mathrm{~h}$ during the experiment. The eyes were treated with $1 \%$ atropine sulfate solution to dilate the pupils and with a topical antibiotic (gentamicin sulfate, $3 \%$ ) before being covered with gas-permeable contact lenses. Foveae were mapped onto a tangent screen using a reversing ophthalmoscope. The visual receptive fields of isolated neurons were later mapped on the same tangent screen, keeping reference to the foveae. Proper refraction was achieved by placing corrective lenses in front of the eyes on custom-designed lens holders. The eyes were stabilized with ophthalmic rings to minimize drift of visual field position.

Electrophysiological recordings and data acquisition. A matrix of seven independently moveable electrodes (Thomas Recording) was used to record simultaneously from multiple cortical cells in V1. The seven electrodes were arranged in a straight line with each electrode separated from its neighbor by $\sim 300 \mu \mathrm{m}$. The electrode consists of a platinum/tungsten core $(25 \mu \mathrm{m}$ in diameter and $1 \mu \mathrm{m}$ at the tip) covered with an outer quartz-glass shank ( $80 \mu \mathrm{m}$ in diameter), and has an impedance value of 1-4 $\mathrm{M} \Omega$. The multielectrode matrix was precisely positioned before recordings so the tip of the matrix was $\sim 3 \mathrm{~mm}$ above the cortical surface. We first placed the tips of all seven electrodes near the cortical surface and then advanced them in $\sim 100 \mu \mathrm{m}$ steps. This setup allowed simultaneous recordings of multiple neurons within the same cortical layer, all at similar visual eccentricities. Electrical signals from the seven electrodes were amplified, digitized, and filtered $(0.3-10,000 \mathrm{~Hz})$ with a preamplifier (Tucker-Davis Technologies, model number RA16SD) configured for multichannel recording. The Tucker-Davis system was interfaced to a computer (Dell) running a multichannel version of the OPEQ program (designed by Dr. J. A. Henrie, Center for Neural Science, New York University, New York, NY) to acquire both spike and local field potential data. Spike waveforms were discriminated online with custom software (designed by Dr. J. A. Henrie) during the experiment, and were again carefully verified off-line using custom spike-sorting software (designed by Dr. D. Xing, Center for Neural Science, New York University, New York, NY). Strict criteria for single units included fixed shape of the action potential and the absence of spikes during the absolute refractory period. Visual stimuli were generated also with the custom OPEQ program running in a Linux computer (Dell) with a graphics card with Open GL optimization. Data collection was synchronized with the screen refresh to a precision of $<0.01 \mathrm{~ms}$. Stimuli were displayed on a 20 inch monitor (Iiyama HM 204DTA flat Color Graphic Display; pixels, $1024 \times$
768; frame rate, $100 \mathrm{~Hz}$; mean luminance, $59 \mathrm{~cd} / \mathrm{m}^{2}$ ) with the screen viewing distance of $\sim 114 \mathrm{~cm}$. The basic attributes of each cell were estimated using small drifting sinusoidal gratings surrounded by gray background (both the gratings and the gray background had a mean luminance of $59 \mathrm{~cd} / \mathrm{m}^{2}$ ).

Visual stimuli. We used both sparse noise (Jones and Palmer, 1987) and sinusoidal gratings (Ringach et al., 1997) to map spatiotemporal receptive fields of V1 neurons. The sparse noise (Jones and Palmer, 1987) consisted of a sequence of randomly positioned (in a $12 \times 12$ sample grid) dark and bright squares $\left(0.2^{\circ} \times 0.2^{\circ}\right)$ against a gray background (luminance, $59 \mathrm{~cd} / \mathrm{m}^{2}$ ). The luminance of bright and dark squares was adjusted so the contrasts from the light increment (luminance, $107 \mathrm{~cd} /$ $\mathrm{m}^{2}$ ) and light decrement (luminance, $11 \mathrm{~cd} / \mathrm{m}^{2}$ ) were equal. The stimulus was sparse in both space and time: only one square (either bright or dark) was shown in each frame. Each sparse noise image appeared for 40 or $50 \mathrm{~ms}$ and the entire sequence lasted $\sim 14$ or $\sim 18 \mathrm{~min}$ (a total of 288 images, each image presented 72 times). The grating stimuli (Ringach et al., 1997) were derived from a low-pass subset of the two-dimensional Hartley functions (Bracewell, 1983) that consisted of an orthogonal set of stationary sinusoidal gratings $\left(2.4^{\circ} \times 2.4^{\circ}\right)$ with evenly spaced orientations, spatial phases $(n=4)$ and spatial frequencies $(0.5-8.0$ cycles/deg in visual angle). Each grating image appeared for $20 \mathrm{~ms}$ and the entire sequence lasted $\sim 15 \mathrm{~min}$ (a total of 1368 images, each image presented $\sim 33$ times). The mean luminance of each grating image is equal to the background luminance of the sparse noise (i.e., $59 \mathrm{~cd} / \mathrm{m}^{2}$ ).

Histology. Cells were assigned to different layers of V1 based on the results of track reconstruction (Hawken et al., 1988; Ringach et al., 2002). Along each track, we recorded the depths of every recording site during the experiment, and then made 3-4 electrolytic lesions at 600-900 $\mu \mathrm{m}$ intervals at the end of the experiment (see Fig. $2 \mathrm{~A}$ ). A lesion was made by passing a $3 \mu \mathrm{A}$ DC current for $2 \mathrm{~s}$ (Xing et al., 2005) through the quartz platinum/tungsten microelectrodes (Thomas Recording) with a stimulus generator (ALA Scientific Instruments; model number STG-1001). After the animal was killed, it was perfused through the heart with $1 \mathrm{~L}$ of heparinized saline $(0.01 \mathrm{M}$ PBS) followed by $2-3 \mathrm{~L}$ of fixative $(4 \%$ paraformaldehyde, $0.25 \%$ glutaraldehyde in $0.1 \mathrm{~m}$ phosphate buffer). After the brain was blocked and sectioned at $50 \mu \mathrm{m}$, the lesions were initially located in unstained sections and then the lesion sections were stained for cytochrome oxidase. Cytochrome oxidase provides good anatomical localization of the laminar boundaries. Cortical layers were determined based on the cell density and cytochrome oxidase-specific labeling (Wong-Riley, 1979). After locating the lesions within the sections, we reconstructed the electrode penetration using a camera lucida, and determined the location of each recorded site relative to the reference lesions and the layers of the cortex. Our estimate of cortical depths of recording sites was quite precise: the difference between the estimated unit distance between lesion sites and the physical unit distance between two parallel electrodes (which was relatively constant across layers, see Fig. $2 A$ ) was $<5 \%$ of the average unit distance from the two measures. Therefore, factors such as the shrinkage of the brain section would not affect our estimates substantially. The mean thickness of each layer was then used to determine each cell's normalized cortical depth. The assignment of cells to layers is crucial since the cortical connectivity of different layers in the primate cortex is very different and important for their function (Rockland and Lund, 1983; Lund, 1988).

\section{Results}

We measured spatiotemporal maps of neurons in macaque V1 by means of the reverse correlation method with randomly positioned bright or dark squares as stimuli (Jones and Palmer, 1987). Both bright and dark squares were shown against a gray background; increment (white on gray) and decrement (black on gray) had equal luminance contrast. Figure $1 A$ represents the spatiotemporal responses of an example V1 cell, in which responses to bright and dark squares are shown separately as color maps at a series of stimulus-response time delays (top, increment responses plotted in red; bottom, decrement responses in blue). 

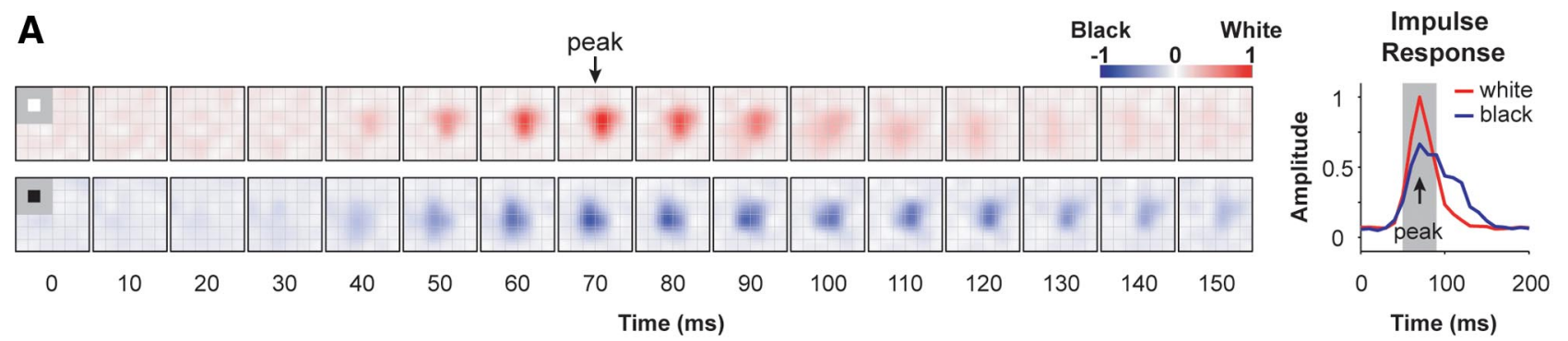

B

Layer-4c examples

C Layer-2/3 examples

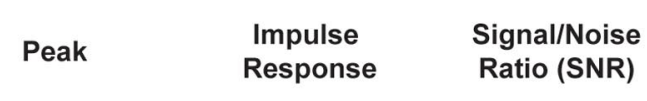

Cell 1
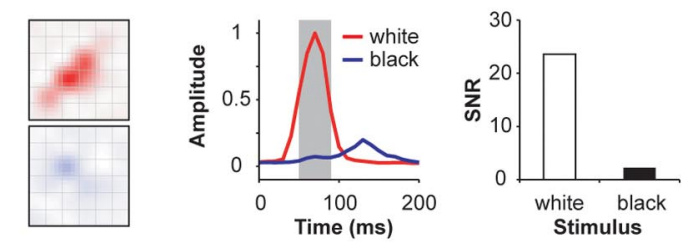

Cell 2
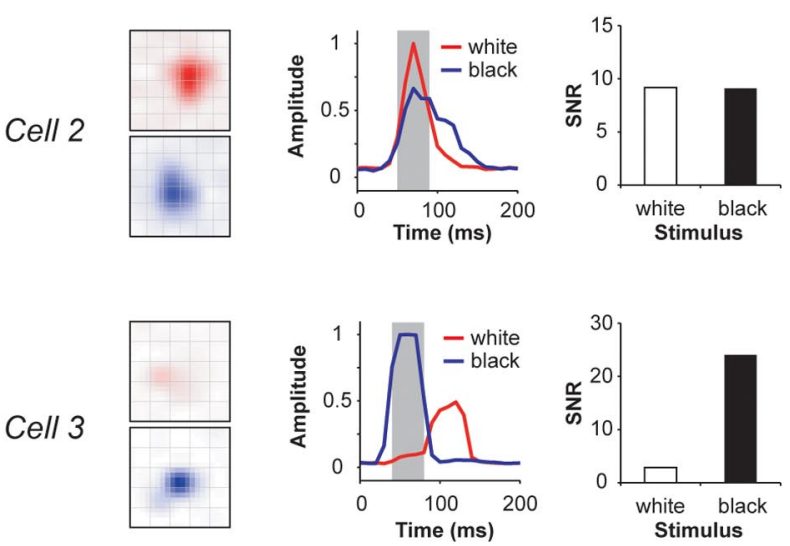

paak
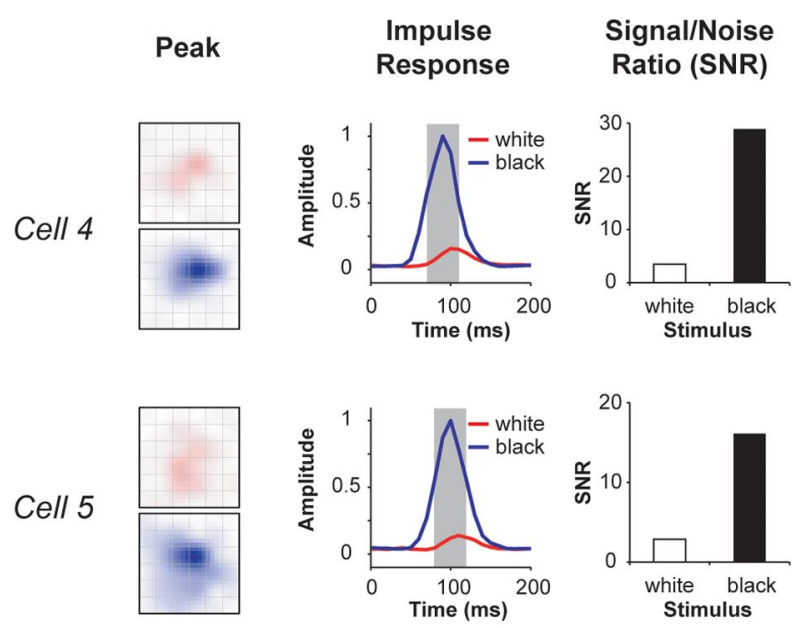

Cell 6
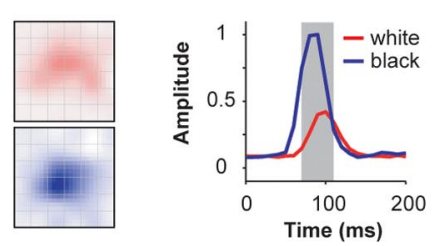

Signal/Noise
Ratio (SNR)

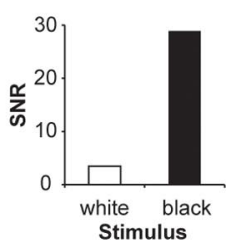

Figure 1. Spatiotemporal maps and impulse responses of V1 neurons. A, Spatiotemporal maps and impulse responses of an example V1 neuron. The spatiotemporal maps are shown separately for increment (a white square on a gray background, top in red) and decrement (a black square on a gray background, bottom in blue) as color maps in a series of time delays between visual stimuli and nerve impulses. The size of each square pixel in the grid was $0.2^{\circ} \times 0.2^{\circ}$ in visual angle, the same size as the individual black and white stimulus squares. The last column represents the response time courses for increment (red) and decrement (blue) responses as the normalized variances of spatial maps at different time delays. $\boldsymbol{B}$, Three example neurons from layer 4 c of V1. C, Three example neurons from layer 2/3 of 1 . For each example, the followings are shown from left to right: the peak spatiotemporal response, the impulse response function, and the SNR for white and black stimuli. The SNR was quantified as the average variance within the peak $40 \mathrm{~ms}$ (gray area) divided by the variance at time offset zero.

Typically, the spatiotemporal response at $0-30 \mathrm{~ms}$ is noise but a signal-locked response emerges at 40-100 ms.

We quantified the relative response to bright squares (ON responses) and to dark squares (OFF responses) by calculating a signal/noise ratio (SNR) based on the spatial energy (or variance) of the response (Ringach, 2002; Malone et al., 2007) as follows. The last column in Figure $1 A$ is a plot of normalized spatial energies of $\mathrm{ON}$ (in red) and OFF (in blue) responses versus the time delay between stimulus and response for the example cell. The time offset at which the spatial energy reached its peak was defined as the "peak time" (arrowed). The SNR was calculated as the ratio between the spatial energy averaged around the peak time (representing signal, gray area in the impulse response figure) and the spatial energy at zero time offset (representing noise in neuron's activity). A cell's response was considered significant if the SNR of either ON $\left(\mathrm{SNR}_{\text {White }}\right)$ or OFF $\left(\mathrm{SNR}_{\mathrm{Black}}\right)$ responses was $>2$.

There was a strong tendency for responses to black stimuli (OFF responses) to be much stronger than responses to white stimuli (ON responses) for the majority of neurons in the output layers $2 / 3$ but not for cells in the input layer $4 \mathrm{c}$. Figure 1, $B$ and $C$, represents the peak spatiotemporal response, the impulse response and the SNR for six example cells, three from layer 4c (Fig. $1 B$ ) and three from layer $2 / 3$ (Fig. $1 C$ ) of V1. The three layer-4c neurons showed different characteristics in responding to black and white stimuli: Cell 1 responded more to white stimuli $\left(\mathrm{SNR}_{\text {White }} / \mathrm{SNR}_{\text {Black }}=23.58 / 2.27\right)$, Cell 2 had nearly equal ON and OFF responses $\left(\mathrm{SNR}_{\text {White }} / \mathrm{SNR}_{\text {Black }}=9.16 / 9.10\right)$, while Cell 3 had a stronger response to black stimuli $\left(\mathrm{SNR}_{\text {White }} / \mathrm{SNR}_{\text {Black }}=\right.$ 2.88/24.05). In contrast, all three layer-2/3 neurons (Cells 4-6) responded more to black than to white stimuli.

The preference for black stimuli in layers $2 / 3$ was evident at the population level. Among a total of 243 single units recorded from different layers of macaque V1, 175 of them had significant responses $\left(\mathrm{SNR}_{\text {White }}\right.$ or $\left.\mathrm{SNR}_{\text {Black }}>2\right)$ and were assigned to different V1 layers through track reconstructions (Fig. 2A; see Materials and Method for details). To determine whether a cell 

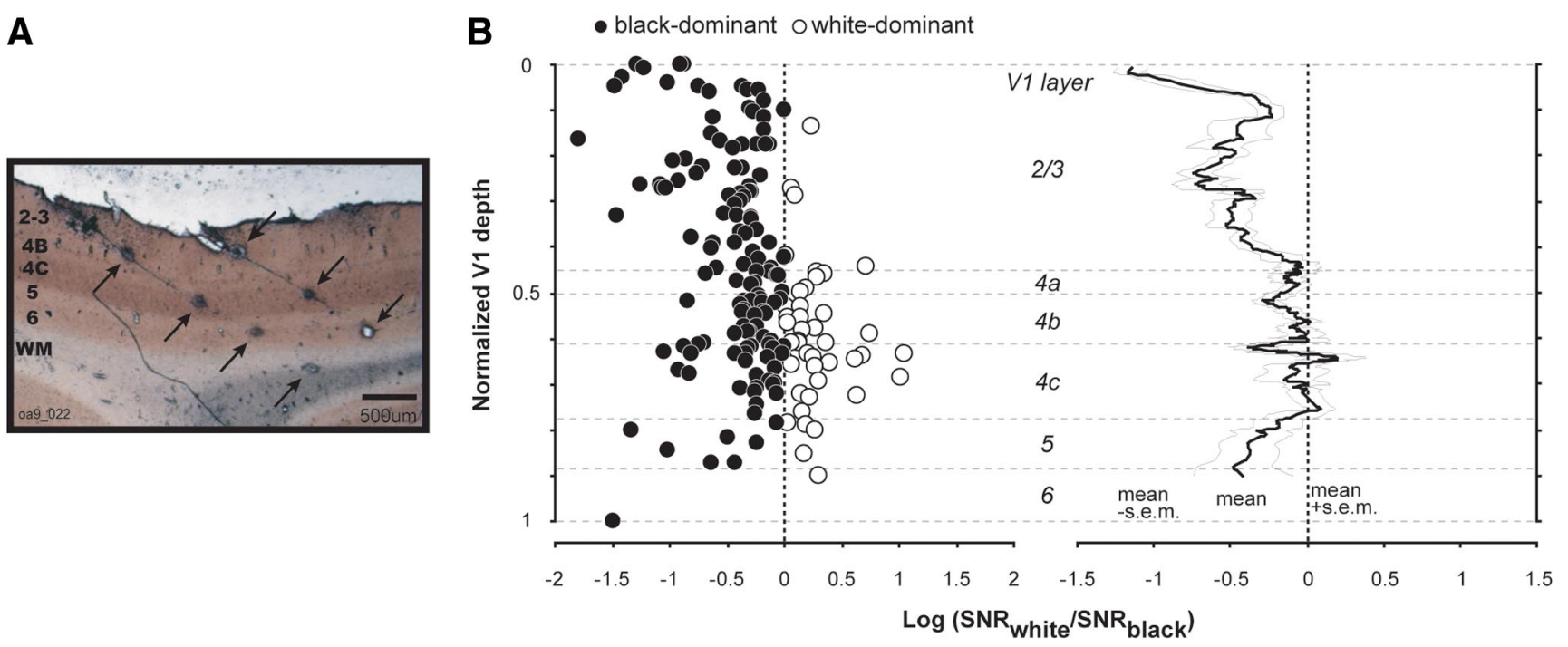

C black-dominant $\square$ white-dominant

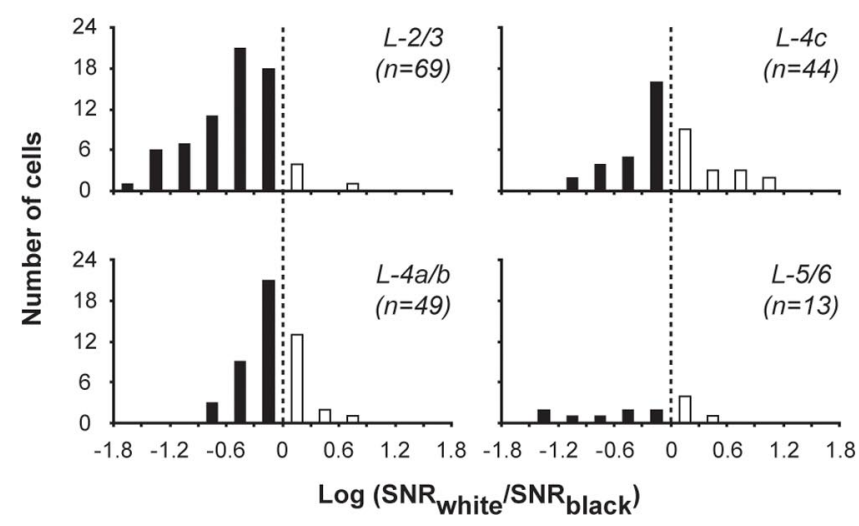

D

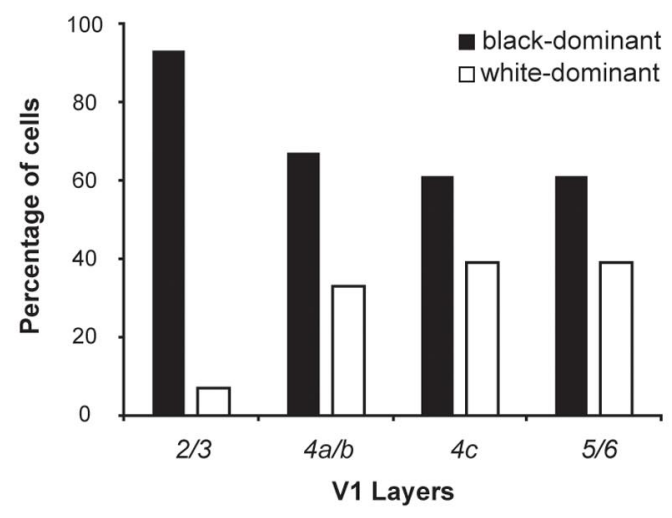

Figure 2. Neurons in layers $2 / 3$ of $\mathrm{V} 1$ respond more to black than to white stimuli. $A, A 50 \mu \mathrm{m}$ cytochrome oxidase-stained brain section with multiple lesions (arrowed) made along 2 recording tracks with quartz platinum/tungsten microelectrodes. $B$, The $0 \mathrm{~N} / \mathrm{OFF}$ ratios $\left[\log \left(\mathrm{SNR}_{\text {white }} / \mathrm{SNR}_{\text {black }}\right)\right]$ of individual neurons (left) and their running averages (right) plotted versus $\mathrm{V} 1$ cortical depth. The running averages (from 9 neighboring cells) represent a smoothed version of the variation of ON/OFF ratio. C, Histograms of the ON/OFF ratios assigned to different layers of V1. $D$, The percentages of black-dominant $(\mathrm{ON} / \mathrm{OFF}$ ratio $<0)$ and white-dominant $(\mathrm{ON} / \mathrm{OFF}$ ratio $\geq 0)$ neurons in different layers of V1. The responses to white and to black were based on results of sparse noise with reverse correlation.

was either ON- or OFF-dominated, we derived an index: $\log \left(\mathrm{SNR}_{\text {White }} / \mathrm{SNR}_{\text {Black }}\right)$, which we called “ON/OFF ratio." The $\mathrm{ON} / \mathrm{OFF}$ ratio is positive if the response to white stimuli is stronger and negative if the response to black stimuli is stronger.

The ON/OFF ratios of individual neurons varied across V1 layers (Fig. $2 B$ ). The average ON/OFF ratio was significantly more negative (meaning more black/OFF dominance) for layer$2 / 3$ neurons (ON/OFF ratio $=-0.51+0.45$, mean $+\mathrm{SD}, n=$ $69)$ than for layer- $4 \mathrm{c}$ cells for which the ON/OFF ratio was close to zero $(\mathrm{ON} / \mathrm{OFF}$ ratio $=-0.07+0.47$, mean $+\mathrm{SD}, n=44)$, and than for layer-4a/b neurons (ON/OFF ratio $=-0.12+0.30$, mean $+\mathrm{SD}, n=49)$. The differences in the ON/OFF ratio between layers- $2 / 3$ and layer- $4 c$ and between layers- $2 / 3$ and layer$4 \mathrm{a} / \mathrm{b}$ neurons were both statistically significant (the probabilities they were drawn from the same distribution were both $p<$ 0.0001 , Wilcoxon rank sum test). The ON/OFF ratios between layer $4 \mathrm{a} / \mathrm{b}$ and layer $4 \mathrm{c}$ were not significantly different $(p=0.44$, Wilcoxon rank sum test). On average, black/OFF responses were $\sim 3 \times$ stronger than white/ON responses in layer-2/3, but only $\sim 20 \%$ stronger for layer- $4 \mathrm{c}$ neurons and $\sim 30 \%$ stronger for layer- $4 \mathrm{a} / \mathrm{b}$ neurons. Overall, the ON/OFF ratio averaged across all V1 layers in our sample was -0.28 , equivalent to an average OFF response that is $90 \%$ larger than ON. A previous investigation in awake monkeys reported a similar but smaller OFF-dominance in color-sensitive V1 neurons (average OFF response 20\% stronger than $\mathrm{ON}$ in terms of firing rates) without any mention of laminar differences (Conway and Livingstone, 2006).

The frequency distributions of ON/OFF ratio also reveal interlaminar differences (Fig. 2C,D). Among neurons that were strongly polarized ( $\mid \mathrm{ON} / \mathrm{OFF}$ ratio $\mid>0.3$, i.e., one polarity is at least twice as strong as the other) (Fig. 2C), the numbers of blackand white-dominant neurons were approximately equal in layer 4c (black/white, 11/8), whereas almost all of those in layer $2 / 3$ were black-dominated (black/white, 46/1). Overall, a large majority of layer-2/3 neurons had stronger black/OFF than white/ON responses $(93 \%, n=64 / 69)$. The percentages of blackpreferring (OFF-dominant) neurons in layer $4 \mathrm{c}(61 \%, n=27 / 44)$ and layer $4 \mathrm{a} / \mathrm{b}(67 \%, n=33 / 49)$ were smaller (Fig. $2 D)$.

These are three possible scenarios for layer- $2 / 3$ neurons to show much stronger black preferences than layer-4c neurons. First, layer-2/3 neurons have stronger responses to black, but their responses to white are the same as those of layer- $4 \mathrm{c}$ neurons. Second, layer-2/3 neurons have weaker responses to white, but their responses to black are the same as those of layer- $4 \mathrm{c}$ neurons. 

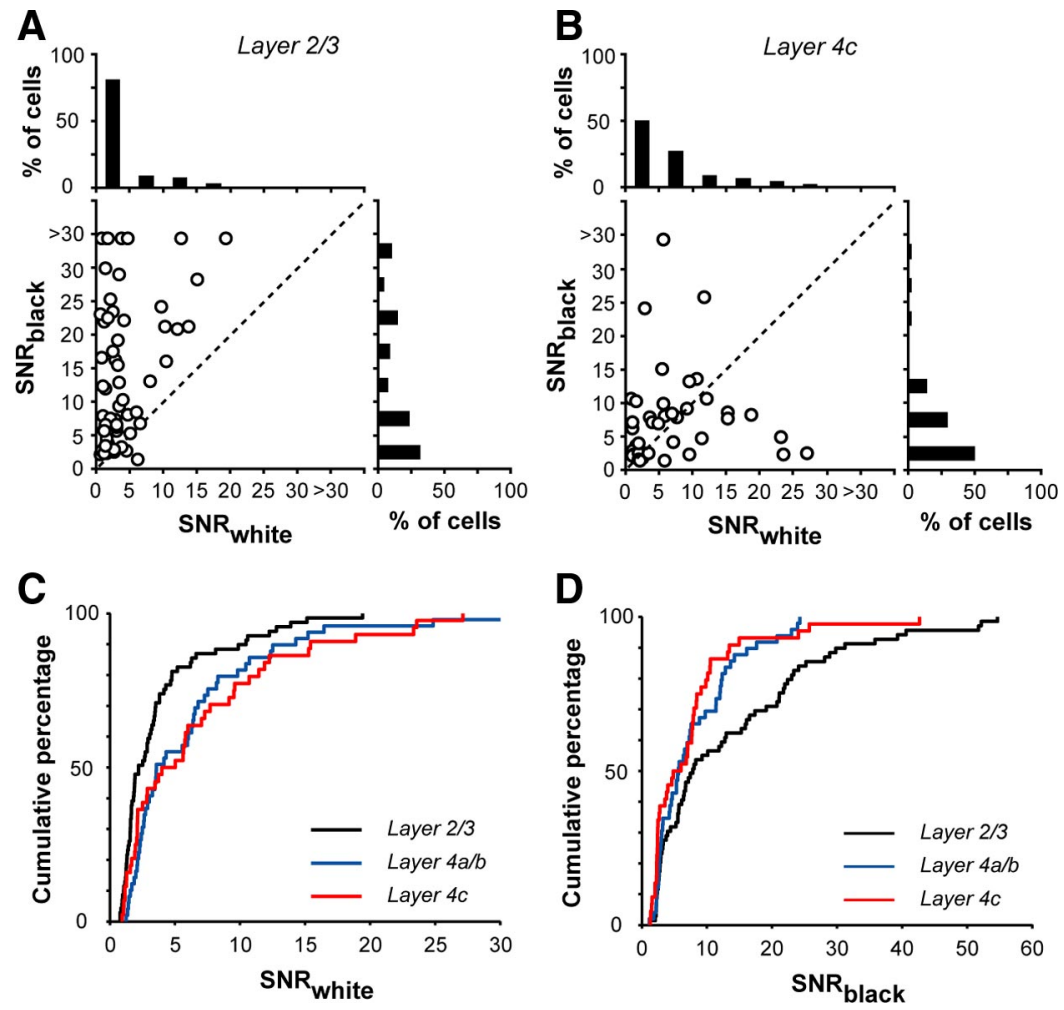

Figure 3. The distributions of bright- and dark-response amplitudes differ significantly between layer- $2 / 3$ neurons and their layer-4 counterparts. $A, B, S$ catter plots and histograms of $S N R_{\text {white }}$ and $S N R_{\text {black }}$ for neurons in different layers of V1. $A$, Layers $2 / 3$; $\boldsymbol{B}$, layer 4c. The diagonal represents equal bright and dark responses. $\boldsymbol{C}, \boldsymbol{D}$, Cumulative percentages of $\operatorname{SNR}_{\text {white }}(\boldsymbol{C})$ and $\operatorname{SNR}_{\text {black }}(\boldsymbol{D})$ for neurons in different $\mathrm{V} 1$ layers. The bright and dark responses were based on results of sparse noise with reverse correlation.

Third, layer-2/3 neurons have stronger responses to black AND weaker responses to white than layer-4c neurons. Our results indicated that the third was the most likely scenario for the black/ OFF dominance of layer-2/3 neurons. Histograms and scatter plots of $\mathrm{SNR}_{\text {White }}$ and $\mathrm{SNR}_{\text {Black }}$ (Fig. $3 A, B$ ) reveal that layer-2/3 neurons respond less to white stimuli $\left(\mathrm{SNR}_{\text {White }}>5,19 \%\right.$ for layer- $2 / 3$, $50 \%$ for layer- $4 \mathrm{c})$ and more to black stimuli $\left(\mathrm{SNR}_{\text {Black }}>5,68 \%\right.$ for layer- $2 / 3,50 \%$ for layer-4c). The shape of the cumulative distributions of $\mathrm{SNR}_{\text {White }}$ and $\mathrm{SNR}_{\text {Black }}$ (Fig. $3 C, D$ ) were significantly different between layer- $2 / 3$ neurons and their layer- 4 counterparts $\left(\mathrm{SNR}_{\text {White: }}: 2 / 3\right.$ vs $4 \mathrm{c}, p=0.008 ; 2 / 3$ vs $4 \mathrm{a} / \mathrm{b}, p=0.002$; $\mathrm{SNR}_{\text {Black }}: 2 / 3$ vs $4 \mathrm{c}, p=0.009 ; 2 / 3$ vs $4 \mathrm{a} / \mathrm{b}, p=0.04$; two-sample Kolmogorov-Smirnov test). Overall, black-preferring/OFF-dominant neurons were more abundant than white-preferring/ON-dominant neurons in macaque V1 $(75 \%, n=132 / 175)$, but the proportions of black- and white-preferring neurons depended strongly on laminar location.

The degree of dark/OFF dominance of layer-2/3 neurons depends strongly on the stimulus ensembles used for receptive-field mapping. Figure $4, A$ and $B$, shows receptive fields of two example cells (layer $2 / 3$ and layer $4 c$, respectively) mapped with two different stimuli. The spatial map of the layer- $2 / 3$ cell shows a strong dark-dominant tendency by revealing a single OFF subregion when mapped with sparse noise (Fig. $4 A$, third row), but shows relatively balanced bright and dark responses by having multiple $\mathrm{ON}$ and $\mathrm{OFF}$ subregions when measured by reverse correlation with sinusoidal gratings (Fig. 4A, fourth row) (Ringach et al., 1997). However, the receptive fields of the example layer-4c cell are very similar under the two stimulus conditions (Fig. 4B) (see also Yeh et al., 2009). Because the bright and dark responses were inseparable under grating stimuli, we derived the ON/OFF ratio by considering only the response amplitudes (AMP) of the strongest ON and OFF pixels within the peak $40 \mathrm{~ms}$ of the receptive fields $-\log \left(\mathrm{AMP}_{\text {White }} / \mathrm{AMP}_{\mathrm{Black}}\right)$. We then used the $\log \left(\mathrm{AMP}_{\text {White }} / \mathrm{AMP}_{\text {Black }}\right)$ as the ON/OFF ratio for both stimulus conditions for the analysis in Figure 4. Among the 175 neurons with significant sparsenoise responses, we only included cells with significant signal/noise ratios under both stimulus conditions $\left(\mathrm{SNR}_{\text {grating }}>\right.$ $1.8, \mathrm{SNR}_{\text {white }}$ or $\mathrm{SNR}_{\text {black }}>2.0, n=81$ ). The $\log \left(\mathrm{AMP}_{\text {White }} / \mathrm{AMP}_{\text {Black }}\right)$ is highly correlated with the $\log \left(\mathrm{SNR}_{\text {White }} / \mathrm{SNR}_{\text {Black }}\right)$ $(r=0.88, p<0.001$, data not shown) for the same neurons under the sparse noise condition, though the latter measure tends to be larger possibly because it considers the response of the entire receptive field rather than the response of a single pixel.

The black preference of layer-2/3 neurons was evident when small and sparse stimuli were applied, but was much weaker when responses were measured with sinusoidal gratings (Fig. $4 C, D$ ). The amplitude of the black/white imbalance measured with sparse noise was significantly more negative than that measured with grating stimuli for layer-2/3 neurons (ON/OFF ratio, sparse noise: $-0.36+$ 0.29 , grating: $-0.07+0.23, p<0.0001$, Wilcoxon signed rank test, $n=27)$. A similar but smaller discrepancy in the ON/OFF ratio under the two stimulus conditions was also found in layer $4 \mathrm{c}(\mathrm{ON} / \mathrm{OFF}$ ratio, sparse noise: $-0.07+0.38$, grating: $0.10+0.37, p=0.02$, Wilcoxon signed rank test, $n=$ 28). Consistently, the correlation of the ON/OFF ratios measured with sparse noise and with gratings was weaker for layer- $2 / 3$ neurons than for layer-4c neurons (layer $2 / 3: r=0.44, p=0.02$; layer $4 c: r=0.62, p=0.0004$ ) (see Fig. $4 E, F$ ). The differences between layer $2 / 3$ and layer $4 \mathrm{c}$ in ON/OFF ratio remain statistically significant under both stimulus conditions - the ON/OFF ratio is significantly more negative for layer- $2 / 3$ neurons than for layer- $4 \mathrm{c}$ neurons (grating: $p=0.005$; sparse noise: $p=0.003$, Wilcoxon rank sum test). Overall, these results indicate that the response properties of V1 neurons, especially those in the superficial layers, are highly dynamic and the degree of the black/white imbalance may depend strongly on different stimulus conditions (David et al., 2004; Sharpee et al., 2006; Victor et al., 2006; Yeh et al., 2009).

\section{Discussion}

The preponderance of black-preferring responses in macaque V1 is consistent with studies in human V1 that revealed a tendency for perception to favor black stimuli. Stimuli with negative contrasts (dark squares on a gray background) induced stronger visual evoked potentials in human V1 than stimuli with positive contrasts (bright squares on a gray background); the black/white imbalance was independent of the mean luminance level (Zemon et al., 1988) and was evident in both adults and children (Zemon et al., 1995). Furthermore, BOLD signals in functional magnetic resonance images (fMRI) were stronger when human observers viewed images with positive skewness (net negative contrast) 
A Layer $2 / 3$ example

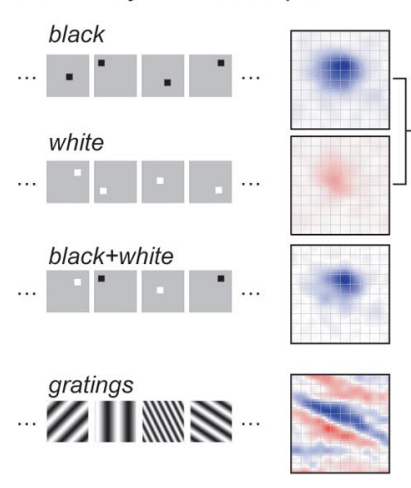

B Layer 4c example

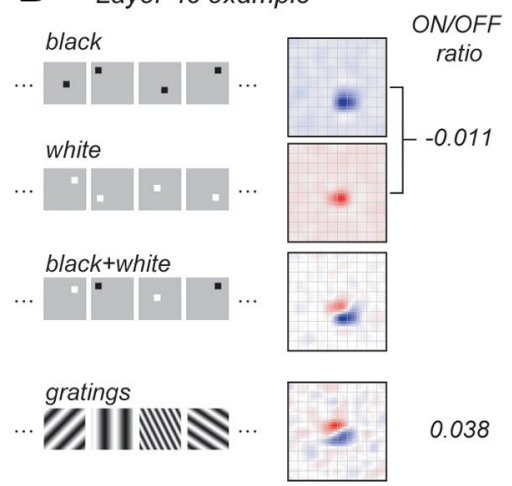
ON/OFF ratio

$-0.078$
C

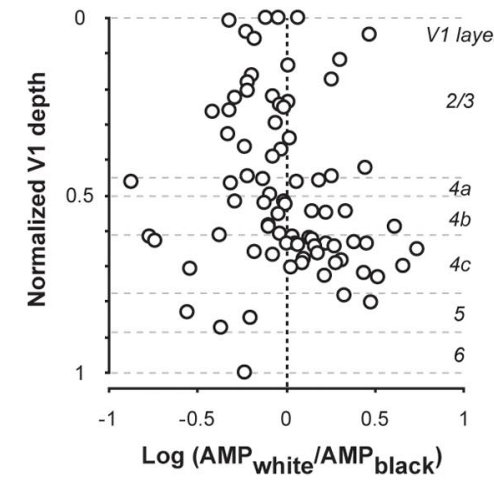

D

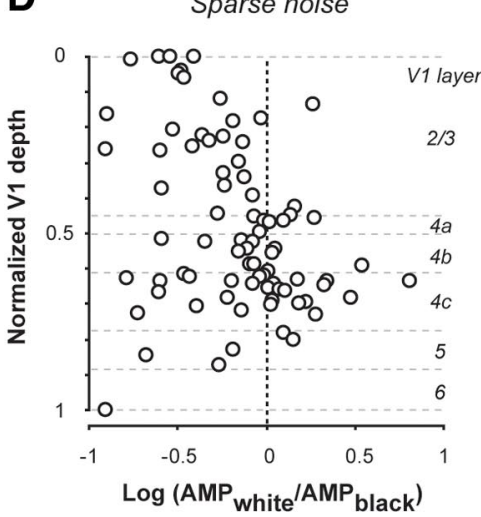

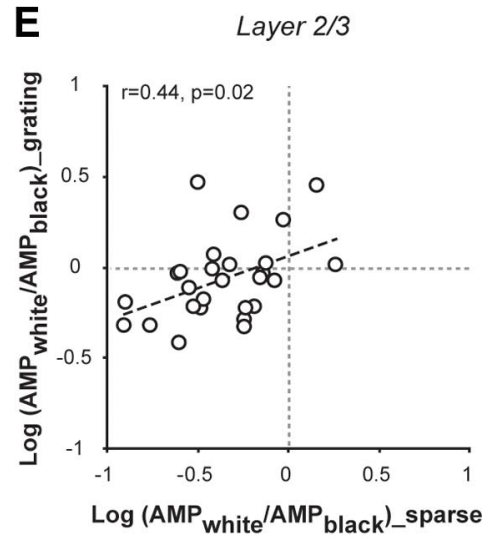

$\mathbf{F}$

Layer $4 c$

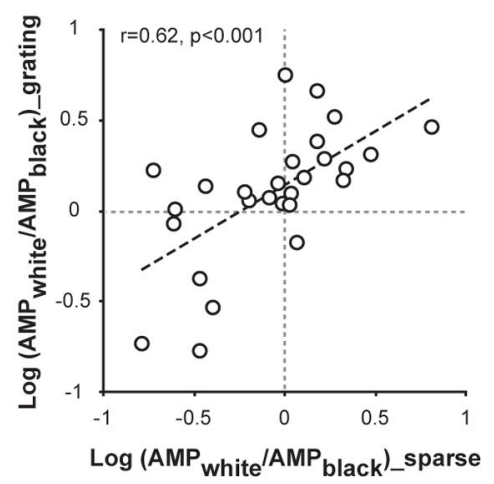

Figure 4. The amplitudes of OFF dominance differ significantly under two different stimulus conditions. $A, B$, Receptive fields of example cells under different stimulus conditions: (from top to bottom) the response to black spots, the response to white spots, the linear summation of responses to black spots and to white spots, and the response to sinusoidal gratings. $\boldsymbol{A}, \mathbf{L a y e r s} 2 / 3 ; \boldsymbol{B}$, layer 4c. C, The $0 \mathrm{~N} / \mathrm{OFF}$ ratios $\left[\log \left(\mathrm{AMP}_{\text {white }} / \mathrm{AMP}_{\text {black }}\right)\right]$ of individual neurons mapped with sinusoidal grating stimuli versus $\mathrm{V} 1$ cortical depth. $\boldsymbol{D}$, The $0 \mathrm{~N} / \mathrm{OFF}$ ratios $\left[\log \left(\mathrm{AMP}_{\text {white }} / \mathrm{AMP}_{\text {black }}\right)\right]$ of individual neurons mapped with sparse noise versus $\mathrm{V} 1$ cortical depth. The $0 \mathrm{~N} / 0 \mathrm{FF}$ ratios for $C$ and $D$ were based on the response magnitudes of the strongest $0 \mathrm{~N}$ and $0 \mathrm{FF}$ pixels within the peak $40 \mathrm{~ms}$ of the spatiotemporal receptive fields. $\boldsymbol{E}$, The $\mathrm{ON} / \mathrm{OFF}$ ratios from the two stimulus conditions were weakly correlated for layer $2 / 3$ neurons. $\boldsymbol{F}$, The $0 \mathrm{~N} / 0 \mathrm{FF}$ ratios from the two stimulus conditions were tightly correlated for layer $4 \mathrm{c}$ neurons.

than when they viewed images with negative skewness (net positive contrast) (Olman et al., 2008). Moreover, the black/white imbalance may also affect visual perception. An image with positive skewness tends to appear darker and glossier than an image with negative skewness for human observers (Motoyoshi et al., 2007). A greater sensitivity for detecting negative contrast may enhance the ability to perceive positive image skewness. Notice that the black/white imbalance we found in macaque $\mathrm{V} 1$ is much larger in layers $2 / 3$ than in the whole $V 1$ population that was measured in the human V1 experiments with fMRI and VEPs (Zemon et al., 1988; Olman et al., 2008).

The preference for black stimuli in V1 may provide the substrate for the advantage of targeting black stimuli that has been found in human psychophysical studies. Human observers' thresholds for detecting decrements tended to be lower than that for increment stimuli (Blackwell, 1946; Short, 1966; Krauskopf, 1980; Whittle, 1986; Bowen et al., 1989). Furthermore, contrast discrimination is better for negative contrast stimuli (Chan and Tyler, 1992; Kontsevich and Tyler, 1999). Reading black-onwhite texts (negative contrasts) is more error-free and faster than reading white-on-black texts (Bauer and Cavonius, 1980; Buchner and Baumgartner, 2007).

The dark/black preference in V1 may also serve as the neuronal substrate for the blackshot mechanism proposed by Chubb et al. (2004). Chubb et al. (2004) found that the discrimination of two texture stimuli is most sensitive when the texture element contrast is the most negative (blackest); the sensitivity of the discrimination is flat as the texture contrast becomes more positive. Moreover, judgments of texture contrast seem to be exclusively determined by the frequency in the texture pattern of lowluminance (lower than the mean luminance) pixels (Chubb and Nam, 2000). Therefore, our findings that most layer- $2 / 3$ neurons in V1 are more sensitive to dark/black stimuli bear a nice relation to the blackshot mechanism and to perception of texture contrast in human psychophysics.

\section{Possible neuronal mechanism for dark-dominant V1}

What is the neural mechanism responsible for the blackpreference of upper layer cells in primate V1? It is unlikely that the profound black/white imbalance in the superficial layer of V1 is inherited from the response properties of neurons in early stages of visual processing. Some previous studies reported that OFF-center cells had narrower dendritic fields (and correspondingly smaller receptive fields) and slower kinetics than ON-center cells in the retina and the visual thalamus (Lankheet et al., 1998; Chichilnisky and Kalmar, 2002). While the asymmetry in receptive field properties between OFF- and ON-center cells might contribute to higher visual acuity in detecting light decrements (Westheimer, 2003) and longer time in detecting temporal order of light-decrement objects (Wehrhahn and Rapf, 1992), it is unlikely to be responsible for stronger black responses in V1-there was no significant asymmetry in response magnitude between 
OFF- and ON-center cells in early visual processing (Krüger and Fischer, 1975; Kremers et al., 1993; Benardete and Kaplan, 1997; 1999) (though Krüger and Fischer, 1975 and Chichilnisky and Kalmar, 2002 found ON-center cells have higher firing rates than OFF-center cells in the retina). Consistent with these studies of subcortical visual processing, here we found the mean ON/OFF ratio in the main thalamic-recipient layer of V1 (layer 4c) was close to 0 (Fig. $2 B$ ), indicating roughly equal strengths of OFF and $\mathrm{ON}$ responses in layer $4 \mathrm{c}$.

The finding of no dark/bright asymmetry in the input layer $4 \mathrm{c}$ of V1 also suggests that a logarithmic luminance transform in early visual processing is unlikely to be the source of the blackdominant responses in layer $2 / 3$ of V1. Our study was conducted around an operating point of the typical luminance range of the CRT monitor (mean luminance, $59 \mathrm{~cd} / \mathrm{m}^{2}$ ). Under these illumination conditions, retinal automatic gain controls cause light adaptation and in this way prevent response compression (for review, see Shapley and Enroth-Cugell, 1984). Retinal light adaptation provides a sufficient explanation why, around an operating point, the input to the cortex should be approximately similar in amplitude for light increments and decrements, as is observed. Therefore, the profound black/OFF-dominance reported here must have been initiated within V1 rather than inherited from subcortical inputs.

It is possible that the preference for black stimuli in adult $\mathrm{V} 1$ is the residual of OFF-dominance early in development. In cat V1 during the first postnatal week, OFF-dominant neurons largely outnumber ON-dominant neurons $(\sim 10: 1)$ in the main thalamic-recipient layers (layers 4 and 6) (Albus and Wolf, 1984).

Another possibility is that the small black/white imbalance in the input layer $4 \mathrm{c}$ is amplified in the superficial layers. We found that neurons that preferred black stimuli slightly outnumbered white-preferring neurons in layer 4c of V1 (27/44 were blackdominant, from Fig. 3 B, C) (see also Jin et al., 2008), possibly as a result of the larger divergence of thalamic afferents of OFF-center cells (Humphrey et al., 1985; Jin et al., 2008; but see Blasdel and Lund, 1983). Layer-2/3 neurons receive feedforward input primarily from layer $4 \mathrm{c}$. The slightly greater abundance of blackpreferring signals in layer $4 \mathrm{c}$ might be amplified in layers $2 / 3$ by recurrent excitation from neighboring black-preferring layer- $2 / 3$ neurons, or by recurrent excitation-inhibition loops in layers $2 / 3$.

\section{Functional significances of introducing dark/bright imbalance within V1}

The finding that the black preference is initiated or greatly amplified within V1 raises an interesting question: what is the functional significance of introducing the black/white imbalance at a post-input cortical stage rather than in earlier visual processing? One possibility is that black and white signals from the retina are kept roughly balanced so that balanced black/white signals can reach other visual brain areas. On the one hand, the ON/OFF asymmetry in higher cortical areas driven by layer-2/3 neurons may play an important role in visual perception and detection (Chubb and Nam, 2000; Motoyoshi et al., 2007). On the other hand, balanced $\mathrm{ON}$ and OFF signals in layer $4 \mathrm{c}$ can excite layer- $4 \mathrm{~b}$ neurons that are direction-selective and transmit signals about target motion to extrastriate visual areas V2 and MT. A psychophysical study found no dark/bright asymmetry in detecting motion of high contrast glass patterns (Anstis et al., 2007; Badcock et al., 2005).

Another possibility for the functional significance of introducing the black/white imbalance in V1 is the dependence of black/white asymmetry on stimulus ensemble. The preference for black stimuli in layers $2 / 3$ of V1 was evident when small and sparse stimuli were applied but was weaker when grating stimuli were used. This discrepancy is largely due to the fact that receptive fields of layer-2/3 neurons differ significantly under the two different stimulus conditions (Yeh et al., 2009). Many layer $2 / 3$ cells have receptive fields with multiple elongated $\mathrm{ON}$ and $\mathrm{OFF}$ subregions when mapped with grating stimuli, but reveal only a single, less-elongated subregion that is frequently dominated by OFF/dark when mapped with sparse noise. These results indicate that the response properties of layer- $2 / 3$ neurons of $V 1$ are highly dynamic, and the degree of the black-over-white bias in V1 superficial layer depends strongly on stimulus conditions (David et al., 2004; Sharpee et al., 2006; Victor et al., 2006; Yeh et al., 2009).

Neurons in V1, but not in the visual thalamus, are tightly interconnected with each other and therefore might be more suitable for encoding highly dynamic visual information. The black preference in V1 is likely a result of evolution since it has been found in species that are active most of the daytime, including humans (Zemon et al., 1988) and macaque monkeys. It will be interesting to investigate whether animals that are active at twilight or at night show a preference for white.

\section{References}

Albus K, WolfW (1984) Early post-natal development of neuronal function in the kitten's visual cortex: a laminar analysis. J Physiol 348:153-185.

Anstis SM, Smith DR, Mather G (2007) Luminance processing in apparent motion, Vernier offset and stereoscopic depth. Vision Res 40:657-675.

Badcock DR, Clifford CW, Khuu SK (2005) Interactions between luminance and contrast signals in global form detection. Vision Res 45: $881-889$.

Bauer D, Cavonius CR (1980) Improving the legibility of visual display units through contrast reversal. In: Ergonomic aspects of visual display terminals (Grandjean E, Vigliani E, eds), pp 137-142. London: Taylor and Francis.

Benardete EA, Kaplan E (1997) The receptive field of the primate P retinal ganglion cell, I: Linear dynamics. Vis Neurosci 14:169-185.

Benardete EA, Kaplan E (1999) Dynamics of primate P retinal ganglion cells: responses to chromatic and achromatic stimuli. J Physiol 519: $775-790$.

Blackwell HR (1946) Contrast thresholds of the human eye. J Opt Soc Am 36:624-643.

Blasdel GG, Lund JS (1983) Termination of afferent axons in macaque striate cortex. J Neurosci 3:1389-1413.

Bowen RW, Pokorny J, Smith VC (1989) Sawtooth contrast sensitivity: decrements have the edge. Vision Res 29:1501-1509.

Bracewell RN (1983) Discrete Hartley transform. J Opt Soc Am 73: 1832-1835.

Buchner A, Baumgartner N (2007) Text-background polarity affects performance irrespective of ambient illumination and colour contrast. Ergonomics 50:1036-1063.

Chan H, Tyler CW (1992) Increment and decrement asymmetries: implications for pattern detection and appearance. Soc Inform Display Symp Dig 23:251-254.

Chichilnisky EJ, Kalmar RS (2002) Functional asymmetries in ON and OFF ganglion cells of primate retina. J Neurosci 22:2737-2747.

Chubb C, Nam JH (2000) Variance of high contrast textures is sensed using negative half-wave rectification. Vision Res 40:1677-1694.

Chubb C, Landy MS, Econopouly J (2004) A visual mechanism tuned to black. Vision Res 44:3223-3232.

Conway BR, Livingstone MS (2006) Spatial and temporal properties of cone signals in alert macaque primary visual cortex. J Neurosci 26: 10826-10846.

Dannemiller JL, Stephens BR (2001) Asymmetries in contrast polarity processing in young human infants. J Vis 1:112-125.

David SV, Vinje WE, Gallant JL (2004) Natural stimulus statistics alter the receptive field structure of v1 neurons. J Neurosci 24:6991-7006.

Hawken MJ, Parker AJ, Lund JS (1988) Laminar organization and contrast sensitivity of direction-selective cells in the striate cortex of the Old World monkey. J Neurosci 8:3541-3548. 
Humphrey AL, Sur M, Uhlrich DJ, Sherman SM (1985) Projection patterns of individual X- and Y-cell axons from the lateral geniculate nucleus to cortical area 17 in the cat. J Comp Neurol 233:159-189.

Jin JZ, Weng C, Yeh CI, Gordon JA, Ruthazer ES, Stryker MP, Swadlow HA, Alonso JM (2008) On and off domains of geniculate afferents in cat primary visual cortex. Nat Neurosci 11:88-94.

Jones JP, Palmer LA (1987) The two-dimensional spatial structure of simple receptive fields in cat striate cortex. J Neurophysiol 58:1187-1211.

Kontsevich LL, Tyler CW (1999) Nonlinearities of near-threshold contrast transduction. Vision Res 39:1869-1880.

Krauskopf J (1980) Discrimination and detection of changes in luminance. Vision Res 20:671-677.

Kremers J, Lee BB, Pokorny J, Smith VC (1993) Responses of macaque ganglion cells and human observers to compound periodic waveforms. Vision Res 33:1997-2011.

Krüger J, Fischer B (1975) Symmetry between the visual B- and D-systems and equivalence of center and surround: studies of light increment and decrement in retinal and geniculate neurons of the cat. Biol Cybern 20:223-236.

Lankheet MJ, Lennie P, Krauskopf J (1998) Distinctive characteristics of subclasses of red-green P-cells in LGN of macaque. Vis Neurosci 15:37-46.

Lund JS (1988) Anatomical organization of macaque monkey striate visual cortex. Annu Rev Neurosci 11:253-288.

Malone BJ, Kumar VR, Ringach DL (2007) Dynamics of receptive field size in primary visual cortex. J Neurophysiol 97:407-414.

Motoyoshi I, Nishida S, Sharan L, Adelson EH (2007) Image statistics and the perception of surface qualities. Nature 447:206-209.

Nelson R, Famiglietti EV Jr, Kolb H (1978) Intracellular staining reveals different levels of stratification for on- and off-center ganglion cells in cat retina. J Neurophysiol 41:472-483.

Olman C, Boyaci H, Fang F, Doerschner K (2008) V1 responses to different types of luminance histogram contrast. J Vis 8:345.

Pasupathy A, Connor CE (1999) Responses to contour features in macaque area V4. J Neurophysiol 82:2490-2502.

Reid RC, Alonso JM (1995) Specificity of monosynaptic connections from thalamus to visual cortex. Nature 378:281-284.

Ringach DL (2002) Spatial structure and symmetry of simple-cell receptive fields in macaque primary visual cortex. J Neurophysiol 88:455-463.

Ringach DL, Sapiro G, Shapley R (1997) A subspace reverse-correlation technique for the study of visual neurons. Vision Res 37:2455-2464.

Ringach DL, Shapley RM, Hawken MJ (2002) Orientation selectivity in macaque V1: diversity and laminar dependence. J Neurosci 22:5639-5651.
Rockland KS, Lund JS (1983) Intrinsic laminar lattice connections in primate visual cortex. J Comp Neurol 216:303-318.

Schiller PH (1982) Central connections of the retinal ON and OFF pathways. Nature 297:580-583.

Schiller PH, Sandell JH, Maunsell JH (1986) Functions of the ON and OFF channels of the visual system. Nature 322:824-825.

Shapley R, Enroth-Cugell C (1984) Visual adaptation and retinal gain controls. In: Progress in retinal research, vol. 3 (Osborne NN, Chader GJ, eds), pp 263-346. Oxford: Pergamon.

Sharpee TO, Sugihara H, Kurgansky AV, Rebrik SP, Stryker MP, Miller KD (2006) Adaptive filtering enhances information transmission in visual cortex. Nature 439:936-942.

Sherk H, Horton JC (1984) Receptive field properties in the cat's area 17 in the absence of on-center geniculate input. J Neurosci 4:381-393.

Short AD (1966) Decremental and incremental visual thresholds. J Physiol 185:646-654.

Victor JD, Mechler F, Repucci MA, Purpura KP, Sharpee T (2006) Responses of V1 neurons to two-dimensional hermite functions. J Neurophysiol 95: 379-400.

Wehrhahn C, Rapf D (1992) ON- and OFF-pathways form separate neural substrates for motion perception: psychophysical evidence. J Neurosci $12: 2247-2250$.

Westheimer G (2003) Visual acuity with reversed-contrast charts: I. Theoretical and psychophysical investigations. Optom Vis Sci 80:745-748.

Whittle P (1986) Increments and decrements: luminance discrimination. Vision Res 26:1677-1691.

Wong-Riley M (1979) Columnar cortico-cortical interconnections within the visual system of the squirrel and macaque monkeys. Brain Res 162:201-217.

Xing D, Shapley RM, Hawken MJ, Ringach DL (2005) Effect of stimulus size on the dynamics of orientation selectivity in macaque V1. J Neurophysiol 94:799-812.

Yeh CI, Xing D, Williams PE, Shapley RM (2009) Stimulus ensemble and cortical layer determine V1 spatial receptive fields. Proc Natl Acad Sci U S A 106:14652-14657.

Zemon V, Gordon J, Welch J (1988) Asymmetries in ON and OFF visual pathways of humans revealed using contrast-evoked cortical potentials. Vis Neurosci 1:145-150.

Zemon V, Eisner W, Gordon J, Grose-Fifer J, Tenedios F, Shoup H (1995) Contrast-dependent responses in the human visual system: childhood through adulthood. Int J Neurosci 80:181-201.

Zhou H, Friedman HS, von der Heydt R (2000) Coding of border ownership in monkey visual cortex. J Neurosci 20:6594-6611. 